\title{
The Effects of Unmodified Vermiculite on Photooxidation of Polyamide 11 Nanocomposites under Accelerated UV Test
}

\author{
Mustapha Kaci, ${ }^{\mathrm{a}, \mathrm{b}}$ Walter W Focke, ${ }^{\mathrm{b}}$ Elizabet M. van der Merwe \\ ${ }^{a}$ Laboratoire des Matériaux Polymères Avancés (LMPA), Université de Bejaia 06000, Algeria \\ ${ }^{b}$ Institute of Applied Materials, Department of Chemical Engineering, University of Pretoria, South Africa \\ ${ }^{c}$ Chemistry Department, University of Pretoria, South Africa
}

\begin{abstract}
The effects of unmodified vermiculite (UMVC) clay on the photooxidation of polyamide 11 (PA11) were investigated by accelerated UV test up to $600 \mathrm{~h}$. The clay was incorporated to the polymer at a content ratio of $5 \mathrm{wt}$. \%. The film samples were prepared by melt compounding using cast extrusion process. The study showed that accelerated photooxidation induced changes in the chemical structure of both PA11 and PA11 nanocomposite resulting in the formation of imides and carboxylic acids as the main carbonyl products. Furthermore, the carbonyl index increased linearly in the first 360 $\mathrm{h}$ of exposure indicating a fast oxidation process before decreasing. The stability of the irradiated samples was also evaluated by the onset oxidation temperature (OOT) determined by DSC and the results corroborated well those obtained by FTIR spectroscopy.
\end{abstract}

Keywords: Polyamide 11, vermiculite, nanocomposite, photooxidation, accelerated UV test, degradation. PACS: 61,41 ,

\section{INTRODUCTION}

The study of polymer aging under accelerated UV conditions is difficult due to the complexity of the photooxidation process and the various factors which affect its course [1]. The photo-oxidation process depends mostly on the polymer structure, the processing history, the presence of additives and the environmental conditions [2]. In the family of polyamides, PA11 has attracted considerable interest as it is an important commercial polymer, derived from a renewable resource (castor oil) [3]. PA11 is an engineering thermoplastic, which offers excellent piezoelectricity, a low wear and abrasion and high chemical resistance [4]. Recently, a large number of publications have been devoted to PA11 nanocomposites aiming to improve the mechanical as well as the flame retardancy properties of the polymer with clay nanofillers [5]. Indeed, most of the studies on PA11 nanocomposites focused on the preparation methods and the structure-properties relationships of PA11 filled with different clays or CNT [6,7]. However to the best of our knowledge, no data have been yet reported on the photo-oxidation of PA11/vermiculite bionanocomposites, although the photooxidative degradation is considered as one of the primary sources of damage to polymer materials under ambient conditions [8]. In this work, the objective was to investigate the photooxidation under accelerated UV test of PA11/unmodified vermiculite nanocomposite films through the changes in the chemical structure. The effects of UVMC on the degradation of PA11/UVMC nanocomposite were evaluated by tracking the carbonyl index in comparison with the neat PA11. Moreover, the stability of the samples under accelerated photooxidation was also evaluated by the determination of the onset oxidation temperature (OOT).

\section{EXPERIMENTAL}

PA11 was provided in powder form by Arkema (France) under the grade Rilsan ES Naturelle. The polymer has a melting point of $186^{\circ} \mathrm{C}$. According to the manufacturer, this grade is the only high performance polymer which is $100 \%$ based on renewable resources.

The starting vermiculite (UVMC), grade Superfine $(1 \mathrm{~mm})$ from Palabora mining (South Africa) was obtained from Mandoval Vermiculite Company (South Africa). The thermal exfoliation of vermiculite was achieved by exposing the material for $5 \mathrm{~min}$ to the temperature of $700^{\circ} \mathrm{C}$ in a convection oven. Then, the exfoliation into submicronic and

9th International Conference on "Times of Polymers and Composites"

AIP Conf. Proc. 1981, 020048-1-020048-4; https://doi.org/10.1063/1.5045910

Published by AIP Publishing. 978-0-7354-1697-0/\$30.00 
nanoflakes was accomplished by sonication using high power ultrasound. For more details on the exfoliation procedure, refers to the work of Macheca et al. [6].

UVMC was melt-compounded with PA11 at filler content of 5 wt. \%. For comparison, the neat polymer was also subjected to the same processing conditions. The compounding process was carried out using a Nanjing Only Extrusion Machinery Co. Ltd, (Model TE-30/600-11-40) co-rotating twin-screw laboratory extruder (diameter = $30.0 \mathrm{~mm}, \mathrm{~L} / \mathrm{D}=40: 1$ ) operating at a feed rate of $2 \mathrm{~kg} \mathrm{~h}^{-1}$. The barrel temperature profile ranged from 70 to $230^{\circ} \mathrm{C}$ and the screw speed was set at $27 \mathrm{rpm}$. The extruded materials passed through a cooling water bath system, pelletized, and finally dried in a convection oven at $40^{\circ} \mathrm{C}$ for 4 days.

Film samples were prepared by using a single screw extruder of Model Collin CR 72T, Esberg, Germany. Prior to processing, the pellets of both neat PA11 and PA11 nanocomposite obtained from the twin-screw extruder were again dried in an oven at $105^{\circ} \mathrm{C}$ for $24 \mathrm{~h}$. The single extruder has, as terminal, a calendar characterized by two counter-rotating cylinders that allow the film passing through them still in the plastic state and a third cylinder to direct the output material up to the collecting cylinder. The extrusion was conducted at the following temperatures: $169 / 215 / 230^{\circ} \mathrm{C}$ along the barrel. The screw speed was $35 \mathrm{rpm}$. The average film thickness was ranged between 60 to $70 \mu \mathrm{m}$ depending on the sample formulation.

A UV weathering tester made by the Q-Panel Company (Cleveland, Ohio) and equipped with 6 UVA-340 lamps was used to accelerate the photo-aging of the film samples. These lamps are the best available simulation of sunlight in the short wavelength region between 295 and $365 \mathrm{~nm}$. Film sheets with dimensions of 100x70 mm were cut from neat PA11 and PA11/UMVC nanocomposite and exposed to UV light at $63^{\circ} \mathrm{C}$. Specimens were taken out at regular intervals for testing up to $600 \mathrm{~h}$.

Infrared spectra of PA11 and PA11/UVMC nanocomposite samples were recorded on a PerkinElmer spectrum 100 FTIR spectrophotometer at a resolution of $4 \mathrm{~cm}^{-1}$ and represent averages of 16 scans. All spectra were recorded in absorbance mode in the $4000-600 \mathrm{~cm}^{-1}$ region. The absorption band at $1690 \mathrm{~cm}^{-1}$ was used to evaluate the formation rate of imide groups at different photooxidation times. The carbonyl index was obtained by calculating the change in carbonyl absorption band at $1690 \mathrm{~cm}^{-1}$ from the FTIR spectra at different exposure times, using the spectrum of the starting non-oxidized materials as reference. All absorbencies were normalized by the film thickness according to eq. (1) [9]:

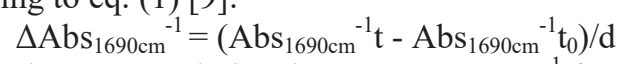

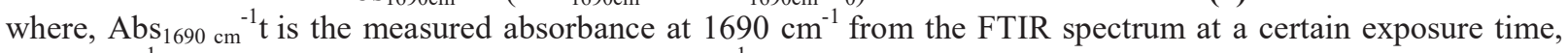

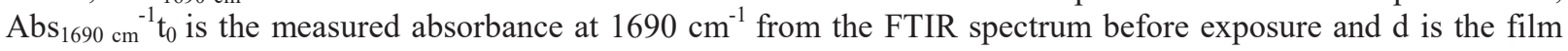
thickness in microns. An average of three replicates was used for each measurement.

The determination of onset oxidation temperature (OOT) is widely used method in the thermal analysis of polymers to evaluate their stability under oxidative conditions [10]. OOT was determined by DSC (DSC1 Star System-Mettler Toledo) and evaluated in accordance to the following procedure: the sample was heated up continuously (i.e. $10^{\circ} \mathrm{C} \mathrm{min}^{-1}$ ) under air flow. OOT was determined as that point in the thermogram where the onset of the decomposition signal resulted. OOT is usually more clearly pronounced as the onset time in oxidation induction time (OIT) measurements.

\section{RESULTS AND DISCUSSION}

Figure 1(a) shows the FTIR spectra of PA11 recorded at different exposure times in the $1800-1680 \mathrm{~cm}^{-1}$ region. A large absorption band is observed in the region $1800-1680 \mathrm{~cm}^{-1}$, whose intensity increases with exposure time, overlapping various carbonyl products. However, two noticeable absorption bands are emerging from this, and growing with exposure time. These previously identified absorption bands located at 1736 and $1690 \mathrm{~cm}^{-1}$ are assigned to imide groups, which are the main photooxidation products. The intensity of these peaks is directly related to the degree of photooxidation of the PA11 matrix. Further, a small absorption band located at $\lambda_{\max }=1713 \mathrm{~cm}^{-1}$ is also noticed, especially at longer exposure time, corresponding to carboxylic acid groups. Accordingly, the photooxidation mechanism of PA11 leads to the formation of imide products resulting from the photochemical decomposition of hydroperoxides through the homolysis of the peroxide bonds. The recombination of the radicals formed leads to imide groups. Similar conclusion can be drawn from PA11/UVMC sample. This is clearly shown in Figure 1(b), related to FTIR spectra of PA11/UVMC (5 wt.\%) nanocomposite. Indeed, the comparison of the different FTIR spectra of PA11 nanocomposite with neat PA11 indicates no shift of the absorption band position in presence of UVMC. This suggests that the photooxidation mechanism of PA11 is not modified in presence of 
UVMC. The accumulation of imide groups with exposure time as a result of hydroperoxide decomposition of neat PA11 and PA11/UVMC nanocomposite was monitored by observing the change in the absorption band at $\lambda_{\max }=1690$ $\mathrm{cm}^{-1}$.
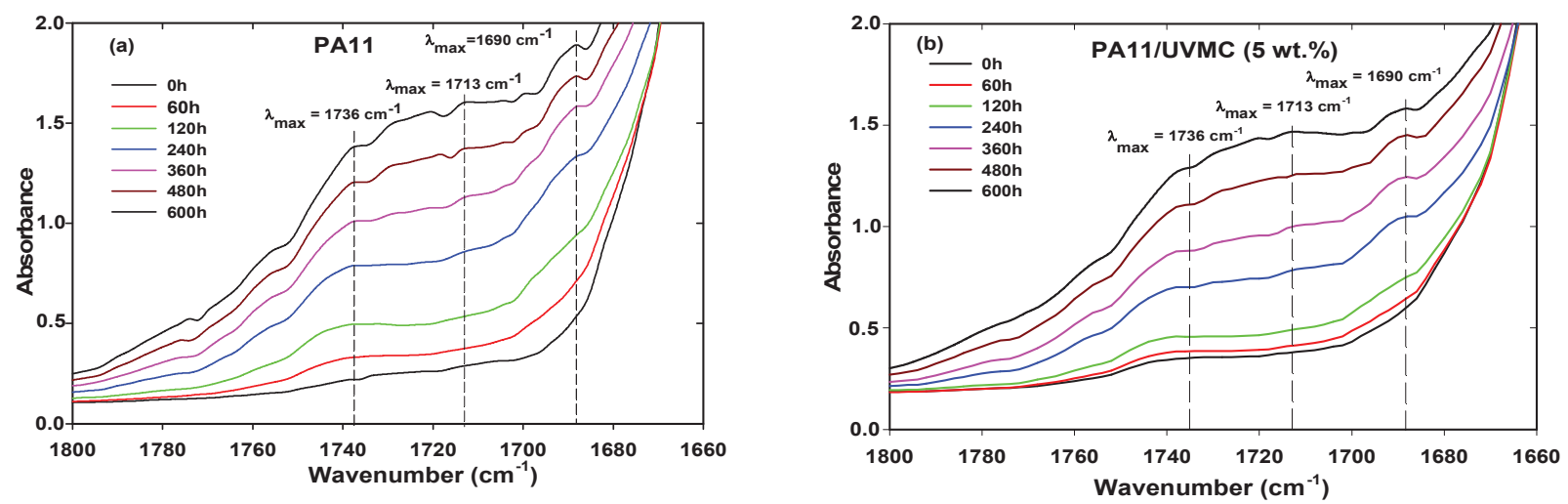

FIGURE 1. FTIR spectra of (a): neat PA11 and (b): PA11/UVMC (5 wt. \%) recorded in the carbonyl region versus time.

Figure 2 compares the photo-oxidation rates through the evolution of $\Delta \mathrm{Abs}_{1690 \mathrm{~cm}^{-1}}$ as a function of exposure time for neat PA11 and PA11/UMVC nanocomposite. The plots show no induction period for carbonyl groups. Furthermore, it is clearly observed that at $300 \mathrm{~h}$, all irradiated samples exhibit a rapid increase in the kinetics curves of $\triangle \mathrm{Abs}_{1690 \mathrm{~cm}^{-1}}$ with slope related to auto-oxidation rate. However at longer exposure time $(>300 \mathrm{~h})$, the photooxidation rate substantially decreases. Figure 2 shows also that the photooxidation rate of PA11 is faster than that of the nanocomposite, although the curves display the same trend, i.e. a hyperbolic shape. Indeed, the unmodified vermiculite exhibits clearly the ability to slow down the photooxidation rate of PA11. One explanation of this surprising result is due probably to the presence of the vermiculite particles at the surface of the film sample, leading to the formation of a layer slowing the oxygen diffusion in the amorphous surface of PA11, thus providing oxygen barrier effects.

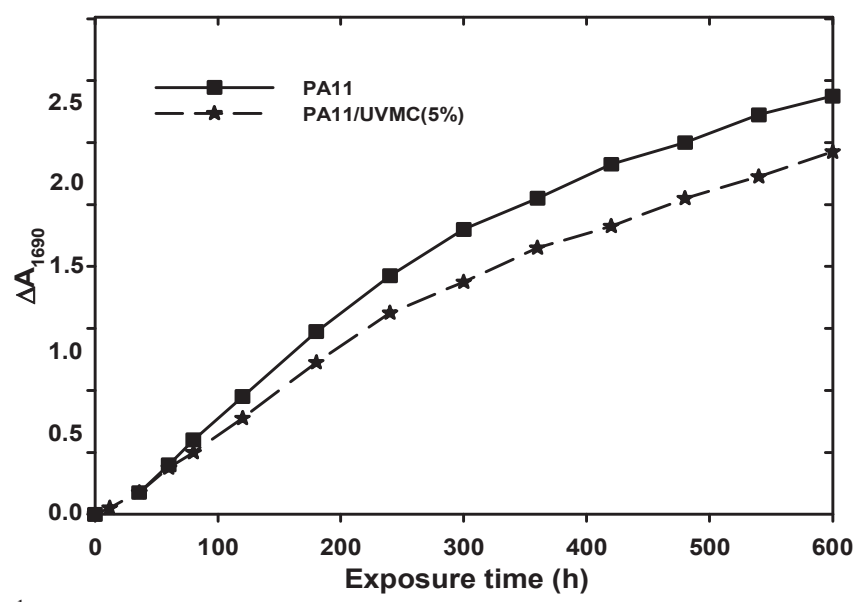

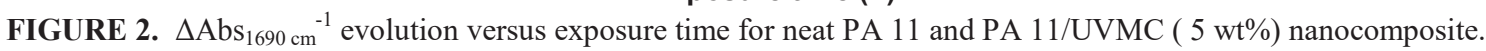

Figure 3 shows the variation of OOT with exposure time for the neat PA11 and PA 11 nanocomposite. It is clearly observed that the PA11/UVMC nanocomposite exhibits an OOT value higher than that of the neat PA11 before exposure. Further, a linear decrease of OOT with exposure time is observed for the whole irradiated samples, however much less lower for the PA 11 nanocomposite. The PA11/UVMC nanocomposite exhibits an initial value of almost $315^{\circ} \mathrm{C}$, while that of neat PA11 is roughly $312^{\circ} \mathrm{C}$. After $600 \mathrm{~h}$ of exposure, the OOT value for the nanocomposite is $309^{\circ} \mathrm{C}$ whereas for PA11, the value decreases to $303^{\circ} \mathrm{C}$. The calculation of the slope values of OOT curves indicates $-1.19810^{-2}$ for the PA11/UVMC nanocomposite against $-2.1510^{-2}$ for the neat polymer. This 
result shows clearly that the addition of unmodified vermiculite to PA11 enhances its resistance against accelerated photo-oxidation, which confirms the data obtained by FTIR

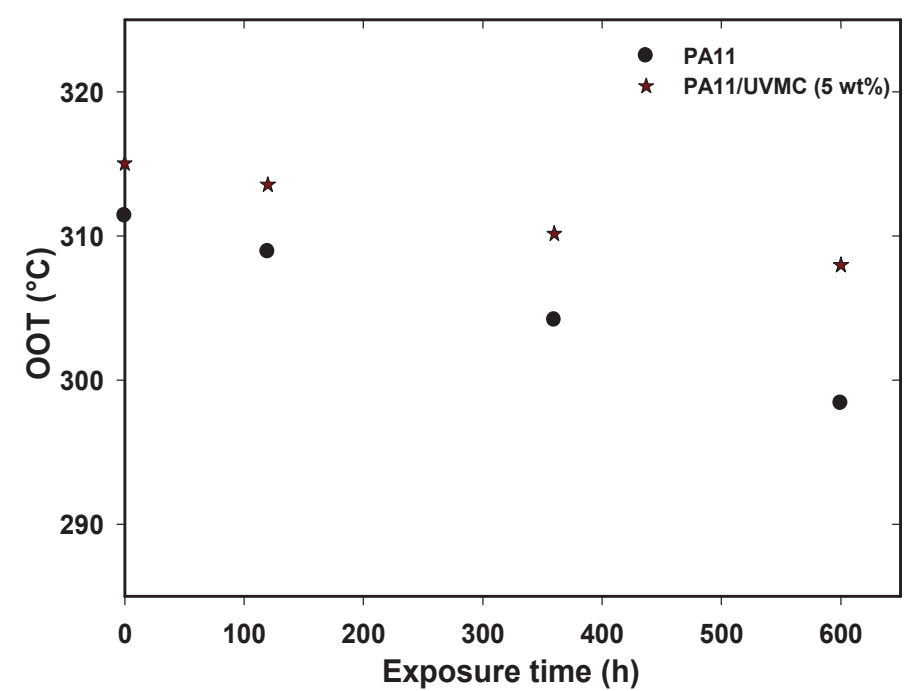

FIGURE 3. OOT evolution as a function of exposure time for neat PA 11 and PA 11/UVMC (5 wt.\%) nanocomposite.

\section{CONCLUSION}

Films based on PA11 and PA11/UVMC (5 wt.\%) were successfully prepared by melt compounding and subjected to accelerated UV test up to $600 \mathrm{~h}$ to evaluate the effect of clay on the photo-oxidation process of the nanocomposite. It was found that the FTIR spectra of the nanocomposite sample exhibited similar shape in the carbonyl region as those of neat PA11in the course of photo-oxidation process characterized essentially by the formation of imides. However, only the absorption band intensity of the carbonyl groups was affected. Indeed, the nanocomposite of PA11/UVMC exhibited better photo-stability than neat PA11 due probably to the accumulation of vermiculite on the film surface leading to the formation of a surface layer acting as oxygen barrier effect, thus reducing the photo-oxidative degradation. This is also confirmed by OOT measurements.

\section{ACKNOWLEDGMENTS}

M.K. would like to thank the DGRSDT-MESRS (Algeria) for its financial support to carry out this study in the frame of the sabbatical year at the University of Pretoria in South Africa.

\section{REFERENCES}

1. M. Kaci, T. Sadoun, K. Moussaceb and N. Akroune, J. Appl. Polym. Sci. 82, 3284-3292 (2001).

2. P. Kiliaris, C. D. Papaspyrides and R. Pfaendner, Polym. Degrad. Stab .94, 389-396 (2009).

3. K. Prashantha, M.-F. Lacrampe and P. Krawczak, J. Appl. Polym. Sci.130, 313-321 (2013).

4. A. Hao, I. Wong, H. Wu, B. Lisco, B. Ong, A. Sallean, S. Butler, M. Londa and J. H. Koo, J. Mater. Sci. 50, 157-167 (2015).

5. G. Mago, D. M. Kalyon and F. T. Fisher, J. Polym. Sci. Part B: Polym. Phys. 49, 1311-1321 (2011).

6. A. D. Macheca, W. W. Focke, H. F. Muiambo and M. Kaci, Eur. Polym. J. 74, 51-63 (2016).

7. S. Huang, M. Wang, T. Liu, W.-D. Zhang, W. Tjiu, C. He and X. Lu, Polym. Eng. Sci. 49, 1063-1068 (2009).

8. E. Olewnik-Kruszkowska. J. Therm. Anal. Calorim. 119, 219-228 (2015).

9. M. Scoponi, S. Cimmino, M. Kaci. Polymer 41, 7969-7980 (2000).

10. M. Schmid, A. Ritter and S. Affolter, J. Therm. Anal. Cal. 83, 367-371 (2006). 